\title{
Sexual signaling and the persistence of alternative reproductive tactics: a test of game theoretic predictions
}

\author{
Courtney R. Garrison a, Scott K. Sakaluk ${ }^{\text {, }}$, Ned A. Dochtermann c, ${ }^{*}$ \\ a Program in Biology, Century College \\ b School of Biological Sciences; Illinois State University \\ c Department of Biological Sciences; North Dakota State University \\ *corresponding author; ned.dochtermann@gmail.com
}

\begin{abstract}
In many species, males produce signals to attract females. However, in some species and populations, only some males produce these signals with other males competing for and "sneaking" reproductive opportunities. In these systems, at least three tactics are expected: always signal, signal only when others are not (assessors), and never signal. The representation of these tactics within a population is unknown in part because the costs of signaling (C) and the fitness value of a single reproductive bout (V) are unknown. Using a game-theoretic model we predict that the always signal strategy only persists if the fitness value of calling is greater than twice the cost. We also show that always signal males are apparently absent in decorated crickets (Gryllodes sigillatus). Moreover, males of this species were not strict assessors and instead signaled infrequently (30\% of the time) when signaling by others was constant. Males also exhibited substantial among-individual variation in the propensity to call when other males were not signaling $(\tau=0.3)$. Our results suggest a high relative cost of signaling $(2 \mathrm{C}>\mathrm{V})$ in this species. The presence of among-individual variation is indicative of underlying genetic variation and a mixed evolutionary stable strategy.
\end{abstract}

Keywords: sexual selection, signaling, game theory 


\section{Introduction}

2 The most dramatic behaviors of animals are often displays associated with attracting mates. Our general understanding of sexual selection provides a robust framework for explaining these varied, and often spectacular, displays observed across taxa. Whether signaling genetic quality or condition (Kokko et al. 2003, Neff and Pitcher 2005), attainment of a specific threshold in quality (Takahashi et al. 2008), or parental ability (Hoelzer 1989), the importance of inter-sexual signaling has led to a diverse range of acoustic, visual, and chemical displays across animal taxa.

However, inter-sexual signals incur costs stemming from both their expression (e.g. energetic costs) and because they may attract predators or otherwise increase the vulnerability of signalers. For example, parasitoids are attracted to the calls of cricket hosts (e.g. Gray et al. 2007), but these calls are necessary to reproduce. Individuals can avoid these costs via the expression of alternative reproductive tactics, such as by remaining silent and intercepting females orienting to signaling males (Cade 1975, Cade 1981, Zuk et al. 2006). In some species and populations, these sneaker individuals dramatically differ from signaling individuals phenotypically, even to the point of being incapable of producing signals (Zuk et al. 2006). In other species and populations, individuals can temporarily adopt either the sneaker or signaling tactics despite the heritability of calling propensity (Cade 1981).

The relative representation of alternative tactics can be estimated based on the relative benefits and costs of alternative tactics and modeled in terms of evolutionary games (Gross 1996). Under game theoretic models (Maynard-Smith 1982), populations are expected to reach equilibrium frequencies of signalers and sneakers, which can include multiple strategies or only one. Here we develop a simple game-theoretic model of when individuals should signal or sneak, and use the predictions of this model to infer the relative costs and fitness benefits of signaling in the decorated cricket (Gryllodes sigillatus).

In the case where males can adopt either a sneaker or signaling strategy three general tactics might be expressed: always signal, never signal, and signal when no other males are signaling (i.e. assess). These strategies carry different costs (C) and fitness benefits (V) depending on which are interacting. We assume that a non-signaling male can intercept a 
33 signal male interacting with either an always signal or assess male will have a payoff of $\frac{1}{4} \mathrm{~V}$,

34 but a payoff of 0 when interacting with another never signal male (Figure 1B). If two

35 individuals with the strategy always signal interact, both will accrue the cost of producing signals but will have an equal probability of mating, leading to a pay-off of $\frac{1}{2} V-C$ (Figure 1B). When an always signal male interacts with either an assess or never signal male, it incurs the cost of signal production $(C)$ and has a 1 in 4 chance of losing a mating opportunity to a sneaker opponent (Figure 1B). When two assess males interact, each has a probability of signaling half of the time, but when interacting with always signal males they have the never signal pay-off. The assess males also have the always signal pay-off when interacting with never signal males. These relationships can be extended across the possible combinations (Figure 1B).

Analysis of this payoff matrix leads to several key predictions (Figure 1C): First, when fitness benefits $(V)$ are less than twice the costs of calling $(C)$, the always signal tactic is predicted to be lost from the population. Second, when fitness benefits are more than twice the costs, never signal individuals are lost from the population. Third, when benefits exceed four times the costs, only the always signal tactic persists. Fourth, when fitness benefits equal twice the costs of calling, all three tactics are expected to be maintained.

These payoffs lead to testable predictions about the composition of populations: if a

51 population contains individuals that always signal, $V \geq 2 \mathrm{C}$. Further, if the population only

52 contains individuals that always signal, then $V>4 C$. If a population contains satellite males

53 that never call, then $V \leq 2 C$. We tested for the presence of each of these tactics in $G$.

54 sigillatus to allow an indirect assessment of $V$ and $C$. The full model description and analysis 55 is presented as supplemental material. 


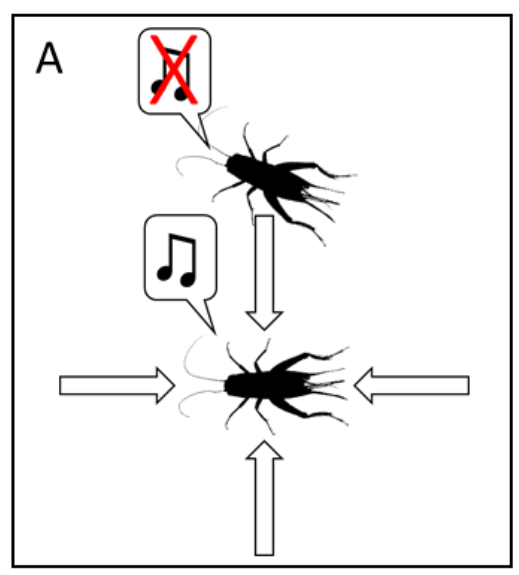

\begin{tabular}{|c|c|c|c|c|}
\hline \multirow[t]{2}{*}{ B } & & \multicolumn{3}{|c|}{ P2 } \\
\hline & & $\begin{array}{c}\text { Always } \\
\text { Signal }\end{array}$ & Assess & Never Signal \\
\hline & $\begin{array}{l}\text { Always } \\
\text { Signal }\end{array}$ & $\frac{1}{2} V-C$ & $V-C-\frac{1}{4} V$ & $V-C-\frac{1}{4} V$ \\
\hline P1 & Assess & $\frac{1}{4} V$ & $\frac{1}{2}(V-C)$ & $V-C-\frac{1}{4} V$ \\
\hline & $\begin{array}{l}\text { Never } \\
\text { Signal }\end{array}$ & $\frac{1}{4} V$ & $\frac{1}{4} V$ & 0 \\
\hline
\end{tabular}



Figure 1. Assumptions, structure, analysis, and predictions from a game theory model of the interactions among individuals who always signal, never signal, and assess. A. Signaling males call (center cricket) and our model assumes females will approach from one of four directions (arrows). Sneaker or satellite males (upper cricket) do not call and, instead, attempt to intercept females. We assume this sneaker male will detect the female first and therefore successfully mate with the female. (pictures courtesy of phylopic.org, Public Domain Mark 1 license) B. Reduced pay-off matrix for each of the tactics when played by individual P1 against another tactic played by $\mathrm{P} 2$. If $\mathrm{V}<2 \mathrm{C}$, the always signal tactic is not expected to persist in the population. If $\mathrm{V}>2 \mathrm{C}$, the never signal tactic is not expected to persist in the population. C. Predicted (above diagonal, bold) and observed (below diagonal) behavioral responses in the playback and clipped trials under two V:C relationships. No always signal individuals were detected in the playback trials, suggesting $\mathrm{V}<2 \mathrm{C}$. However, individuals did not strictly conform to the always or never signal tactics, instead sometimes signaling (middle row). 
Methods

57 We tested these predictions in G. sigillatus from a sampled population descended from individuals captured in New Mexico (USA) and communally housed at high numbers (Ivy et al. 2005). These individuals have been isolated from predation and parasitoids and predators for $\sim 80$ generations. $G$ sigillatus is ideal for testing questions regarding alternative reproductive tactics as males aggregate at high densities in natural populations and switch from signaling to sneaking (S. Sakaluk, pers. obs.). We housed subjects individually in $0.71 \mathrm{~L}$ containers, provided ad libitum food (Purina Chick Starter) and water (provided in glass vials capped with cotton), and provided a shelter made of a small piece of cardboard egg carton. We maintained the cricket housing room at $\sim 27 \mathrm{C}$ and on a 12:12 dark:light cycle reversed such that the room was dark during daytime hours.

To determine if our sample of $G$. sigillatus contained always signal individuals, we tested whether individuals would call in the presence of continual signaling by other males. Specifically, we recorded 20 males during 10 recording bouts (separated by an average of 5 days), with each bout lasting 2 hours. During these bouts, continuous signals of other male G. sigillatus were played such that individuals adopting an assess strategy would not call, differentiating them from always signal individuals. Whether an individual called during these 2 -hour playback bouts was then recorded. If $V<2 C$, the above game-theory model would predict an absence of always signal individuals and that individuals adopting the assess and never signal tactics would not call (Figure 1C).

To determine if never signal individuals were present in our population, we tested whether individuals would call in the presence of a non-focal male that was not calling. We again recorded the same 20 males as used in the playback trials. Each individual was recorded during another 10, 2-hour bouts. To prevent the non-focal male from signaling, we surgically prevented these males from doing so via wing clipping following (Stoffer and Walker 2012). To control for the possibility that the non-focal male identity influenced signaling propensity, all non-focal males were drawn from a population of isogenic $G$. sigillatus (see Ivy et al. 2005 for details regarding the production of isogenic individuals).

84 Whether an individual called during these 2-hour playback bouts was then recorded.

85 Following the model described above, if $V>2 C$, we predicted there would be no never 
Together, the playback and clipped-male testing scenarios and 800 total hours of acoustic monitoring allowed us to distinguish between assess individuals and the other two tactics. These testing scenarios also allowed us to evaluate the relative magnitude of fitness benefits and costs of signaling. To test the predictions of our model we compared the predicted behaviors under different $V$ vs. $C$ conditions (Figure 1D) to the observed behavioral frequency.

We also analyzed the outcomes of these trials using generalized linear mixed effects models. Whether an individual did not or did signal ( 0 versus 1 ) was analyzed as a binary variable with temperature (centered and scaled by its standard deviation), repetition number (1:10), mass (centered and scaled by its standard deviation), and time of day (centered and scaled by its standard deviation) included as fixed effects. Individual ID was included as a random factor and among- and within-individual variances estimated along with unadjusted repeatabilities (Nakagawa and Schielzeth 2010, Dingemanse and Dochtermann 2013). This statistical model was fit using the 1 me 4 package (Bates et al. 2007) in the R computing language. We also estimated the relative contribution of individual identity and the various fixed effects to behavioral variation using the $r p t R$ package (Stoffel et al. 2017).

\section{Results}

\section{Calling propensity vis-à-vis game theoretic expectations}

In the playback bouts we predicted that, if $V<2 C$, there would be no individuals that always signaled (Figure 1D). Indeed, we found that no individuals always signaled. However, contrary to model assumptions, individuals in the playback trials did not wholly abstain from signaling, instead occasionally doing so (Figure 1D).

In the clipped-male bouts, we predicted that there would be no individuals that never signaled if $V>2 C$ (Figure 1D). In contrast, we did find that one individual-over 20 hours of recording-never signaled while in the presence of a clipped-male (Figure 1D). If $V>2 C_{\mathrm{L}}$-we also predicted that all individuals would signal (i.e. both assess and always call individuals would call). Importantly, while males signaled most of the time during clippedmale bouts (75\% of bouts, Figure 2), individuals did refrain from signaling during some bouts (Figure 1D). 

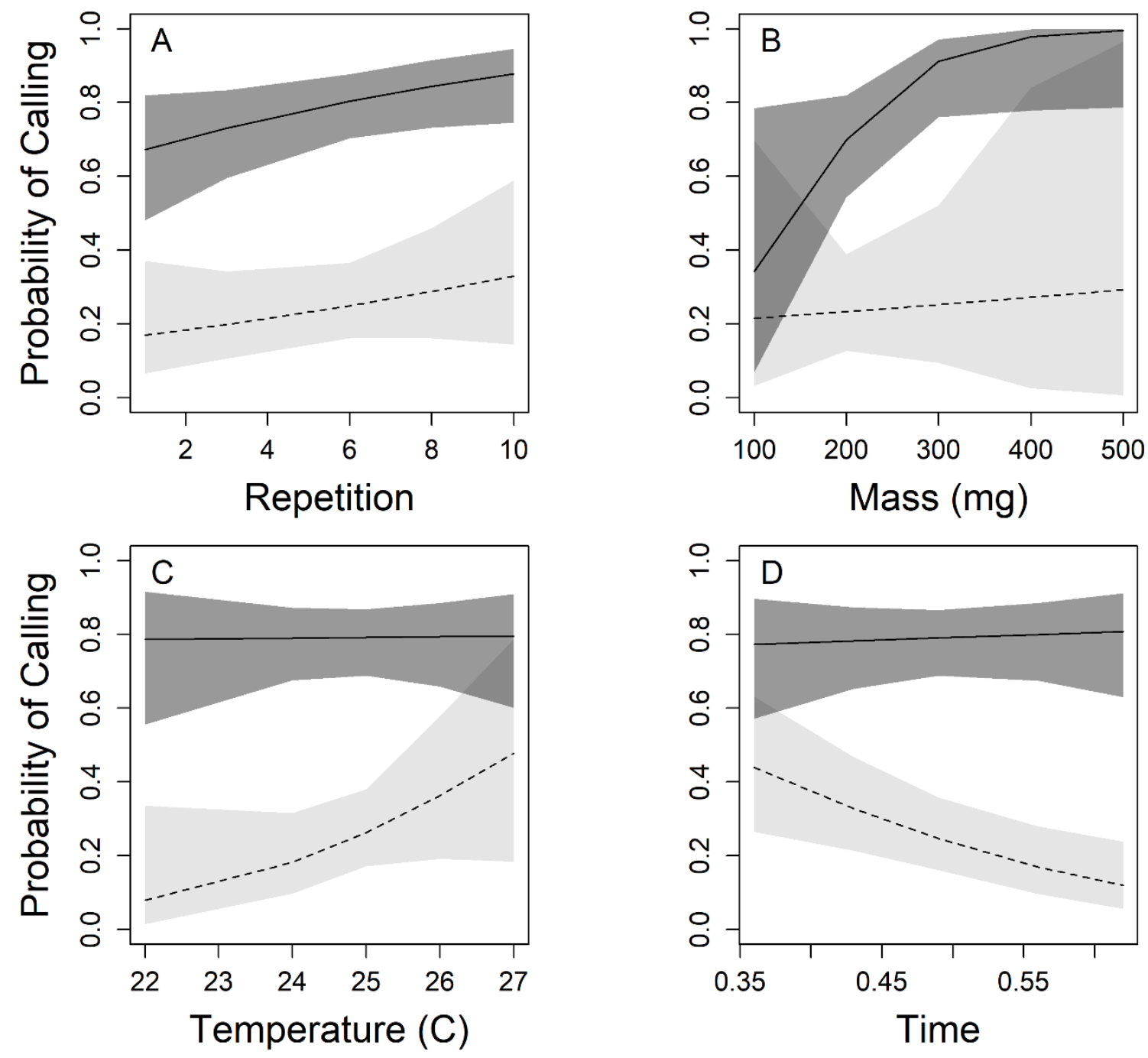

Figure 2. Relationship between probability of calling and (A) Repetition, i.e. over the sequence of recording bouts; (B) Mass, in miligrams; (C) Temperature, in degrees Celsius; and (D) Time of day (scaled from 0 to 1). In all panels the solid lines and dark gray shading corresponds to clipped males. Dashed lines and light gray shading correspond to results from the playback experiment. Shading corresponds to $95 \%$ confidence intervals. 


\section{General calling behavior}

We found that crickets were significantly more likely to signal when in the presence of clipped males than during playback bouts (Figure 2, Table S1): males signaled during 74\% of the clipped-male bouts, versus $35 \%$ of the playback bouts (Table S1; z = -3.07, p = 0.002). Probability of signaling did not significantly change across trials (Figure 2A, Table S1; z = $1.884, \mathrm{p}=0.06$ ) or with temperature (Figure $2 \mathrm{C}$, Table $\mathrm{S} 1 ; \mathrm{z}=0.057, \mathrm{p}=0.95$ ). Propensity to call increased with mass (Figure $2 \mathrm{~B}$, Table S1; $\mathrm{z}=2.002, \mathrm{p}=0.045$ ), a relationship that did not differ by treatment (Table S1). While there was no relationship between time and propensity to call when in the presence of clipped-males (Figure 2D, Table S1; z = 0.27, p = 0.79), during the playback bouts individuals decreased their propensity to call later into the dark period (Figure 2D, Table S1; $\mathrm{z}=-2.12, \mathrm{p}=0.034$ ). Finally, we found that repeatability differed between the playback and clipped bouts. Repeatability during playback was $0.114(\mathrm{p}=0.01)$ whereas it was $0.3(\mathrm{p}<0.001)$ in the clipped-male bouts.

\section{Discussion}

The lack of individuals who always signaled during the playback bouts suggests the absence of always call individuals, though it is possible that they persist at a lower frequency than detectable here. Nonetheless, the combined results of the playback and clipped-male bouts are consistent with the fitness benefits of a single reproductive event being less than twice the costs of signaling (i.e. $V<2 C$ ). This finding is particularly surprising given that this population has been free of selection from predation and parasitoids for $\sim 80$ generations. Consequently, in this population, the costs of signaling should only include metabolic costs.

The energetic costs of calling in G. sigillatus are currently unknown, and these costs are highly variable across gryllid crickets: Hack (1998) found that the metabolic rate in house crickets (Acheta domesticus) while producing advertisement calls was approximately one and a half times an individual's resting metabolic rate. Similarly, Hoback and Wagner Jr (1997) estimated that advertisement calling corresponded to an increase of $\sim 2.2$ times resting metabolic rate in variable field crickets (Gryllus lineaticeps). At the other extreme, calling mole crickets (Gryllotalpa monaka) increase their metabolism 13.5-fold relative to resting rates (White et al. 2008) and, for many crickets, calling similarly requires substantial increases in metabolic rates (e.g. Table 3 in Hack 1998). Based on these varied costs, it is unclear whether the relative costliness of calling in G. sigillatus is due to its 


\section{Acknowledgements}

We thank Tracie Ivy for original collection of crickets and establishing inbred lines. We also thank R. Royauté and H. Sakaluk for important and useful discussions. This work was supported by US NSF IOS grant 1557951 to N.A.D. and NSF IOS grant 1654028 to S.K.S., Ben Sadd, and John Hunt.

\section{References}

171 Bates, D., D. Sarkar, M. D. Bates, and L. Matrix. 2007. The lme4 package. R package version 2:74.

172 Boake, C. R. B. 1989. Repeatability - Its Role in Evolutionary Studies of Mating-Behavior.

$173 \quad$ Evolutionary Ecology 3:173-182.

174 Cade, W. 1975. Acoustically orienting parasitoids: fly phonotaxis to cricket song. Science 190:1312175 1313. 
Cade, W. H. 1981. Alternative male strategies: genetic differences in crickets. Science 212:563-564.

177

178

179

180

181

182

183

184

185

186

187

188

189

190

191

192

193

194

195

196

197

198

199

200

201

202

203

204

205

206

207

208

209

210

Dingemanse, N. J., and N. A. Dochtermann. 2013. Quantifying individual variation in behaviour: mixed-effect modelling approaches. Journal of Animal Ecology 82:39-54.

Dingemanse, N. J., and N. A. Dochtermann. 2014. Individual behaviour: behavioural ecology meets quantitative genetics.in A. Charmantier, D. Garant, and L. E. B. Kruuk, editors. Quantitative genetics in the wild. Oxford University Press.

Dochtermann, N. A., T. Schwab, and A. Sih. 2015. The contribution of additive genetic variation to personality variation: heritability of personality. Proceedings Of The Royal Society BBiological Sciences 282:20142201.

Gray, D. A., C. Banuelos, S. E. Walker, W. H. Cade, and M. Zuk. 2007. Behavioural specialization among populations of the acoustically orienting parasitoid fly Ormia ochracea utilizing different cricket species as hosts. Animal Behaviour 73:99-104.

Gross, M. R. 1996. Alternative reproductive strategies and tactics: diversity within sexes. Trends in Ecology \& Evolution 11:92-98.

Hack, M. A. 1998. The energetics of male mating strategies in field crickets (Orthoptera: Gryllinae: Gryllidae). Journal of insect behavior 11:853-867.

Hoback, W. W., and E. W. Wagner Jr. 1997. The energetic cost of calling in the variable field cricket, Gryllus lineaticeps. Physiological Entomology 22:286-290.

Hoelzer, G. A. 1989. The Good Parent Process Of Sexual Selection. Animal Behaviour 38:1067-1078.

Ivy, T. M., C. B. Weddle, and S. K. Sakaluk. 2005. Females use self-referent cues to avoid mating with previous mates. Proceedings Of The Royal Society B-Biological Sciences 272:2475-2478.

Kokko, H., R. Brooks, M. D. Jennions, and J. Morley. 2003. The evolution of mate choice and mating biases. Proceedings of the Royal Society of London. Series B: Biological Sciences 270:653664.

Maynard-Smith, J. 1982. Evolution and the theory of games. Cambridge University Press, Cambridge.

Nakagawa, S., and H. Schielzeth. 2010. Repeatability for Gaussian and non-Gaussian data: a practical guide for biologists. Biological Reviews 85:935-956.

Neff, B. D., and T. E. Pitcher. 2005. Genetic quality and sexual selection: an integrated framework for good genes and compatible genes. Molecular Ecology 14:19-38.

Stoffel, M. A., S. Nakagawa, and H. Schielzeth. 2017. rptR: Repeatability estimation and variance decomposition by generalized linear mixed-effects models. Methods in Ecology and Evolution 8:1639-1644.

Stoffer, B., and S. E. Walker. 2012. The use of multimodal communication in mate choice decisions by female house crickets, Acheta domesticus. Animal Behaviour 83:1131-1138. 
211 Takahashi, M., H. Arita, M. Hiraiwa-Hasegawa, and T. Hasegawa. 2008. Peahens do not prefer peacocks with more elaborate trains. Animal Behaviour 75:1209-1219.

213 White, C. R., P. G. Matthews, and R. S. Seymour. 2008. In situ measurement of calling metabolic rate in an Australian mole cricket, Gryllotalpa monanka. Comparative Biochemistry and Physiology Part A: Molecular \& Integrative Physiology 150:217-221.

216 Zuk, M., J. T. Rotenberry, and R. M. Tinghitella. 2006. Silent night: adaptive disappearance of a sexual signal in a parasitized population of field crickets. Biology Letters 2:521-524. 


\section{Supplemental Materials}

\section{S1. Model details and analysis}

Table S1. Full generalized mixed-effects model output.

\section{S1. Model details and analysis}

We developed a game theory model to determine the expected relative frequencies of always signal, never signal, and assess tactics within a population. Here we describe the model, starting with a description of the tactics that can be played and their pay-offs followed by the analysis of two tactic games and conclude with invasability analysis in the three tactic game.

\section{Playable tactics}

Three tactics are available to be played: always signal, never signal, and assess. In a two person game each player can play any of the three tactics. Because each game is modeling a single male-male interaction and competition for a mate, pay-offs are determined by the fitness value of a single reproductive bout $(V)$ and the costs of calling $(C)$.

1. The never signal tactic models "sneaker" or "satellite" behavior in which males attempt to intercept a female approaching a signaling male (Figure 1A). This tactic does not incur the cost of calling but cannot attract females in the absence of a signaling male. If an opponent male is signaling, we assume the never signal male can intercept a female approaching only from one of four directions (Figure 1A) and does so before the female can reach the signaling male. If two never signal tactics are played against each other, no female can be attracted and no fitness can be gained. Consequently, the average pay-off for this tactic against a calling male is $\frac{1}{4} \mathrm{~V}$ and 0 against another never signal.

2. The always signal tactic models males that signal regardless of which tactic is being played against. This tactic always incurs the cost, $C$, of signaling. If this tactic is played against another always signal tactic, each has a $50 \%$ chance of successful reproduction. If this tactic is played against a non-calling, sneaker tactic, the calling player will lose one out of four reproductive opportunities but still incur the costs of signaling. The average pay-off for this tactic is therefore $\frac{1}{2} V-C$ when played against another always signal tactic and $V-C-\frac{1}{4} V$ when played against non-signaling opponents.

3. The assess tactic models males who determine if opponents are signaling and do not signal if playing against always signal opponents. The assess tactic will, however, signal if playing against never signal opponents. When played against an always 
signal opponent, the assess tactic has the corresponding pay-off of the never signal tactic. When played against never signal opponents, the assess tactic has the pay-off of an always signal tactic. When assess tactics are played against each other, half the time the signal role is adopted and half the time the sneaker role is adopted. The average pay-off is therefore: $\frac{1}{2}\left(V-C-\frac{1}{4} V\right)+\frac{1}{2}\left(\frac{1}{4} V\right)$ which expands to: $\frac{1}{2} V-\frac{1}{2} C-$ $\frac{1}{8} V-\frac{1}{8} C$ which simplifies to: $\frac{1}{2}(V-C)$.

Combined, these pay-offs can be summarized as (see also Figure 1B):

P2

\begin{tabular}{|c|c|c|c|}
\hline & Always Signal & Assess & Never Signal \\
\hline $\begin{array}{l}\text { Always } \\
\text { Signal }\end{array}$ & $\frac{1}{2} V-C, \frac{1}{2} V-C$ & $V-C-\frac{1}{4} V, \frac{1}{4} V$ & $V-C-\frac{1}{4} V, \frac{1}{4} V$ \\
\hline Assess & $\frac{1}{4} V, V-C-\frac{1}{4} V$ & $\frac{1}{2}(V-C), \frac{1}{2}(V-C)$ & $V-C-\frac{1}{4} V, \frac{1}{4} V$ \\
\hline $\begin{array}{l}\text { Never } \\
\text { Signal }\end{array}$ & $\frac{1}{4} V, V-C-\frac{1}{4} V$ & $\frac{1}{4} V, V-C-\frac{1}{4} V$ & 0,0 \\
\hline
\end{tabular}

A two-strategy game: Always Signal vs. Never Signal

We start by analyzing the case where the only available tactics are always signal and never signal. In this case, the pay-off matrix is:

\section{P2}

Always Signal Never Signal

\begin{tabular}{cc|c|c|}
\cline { 3 - 3 } & $\begin{array}{c}\text { Always } \\
\text { P1 }\end{array}$ & $\frac{1}{2} V-C, \frac{1}{2} V-C$ & $V-C-\frac{1}{4} V, \frac{1}{4} V$ \\
\cline { 3 - 4 } & $\begin{array}{c}\text { Never } \\
\text { Signal }\end{array}$ & $\frac{1}{4} V, V-C-\frac{1}{4} V$ & 0 \\
\cline { 3 - 4 } & &
\end{tabular}

Let $p$ equal the frequency of always signal and $1-p$ be the frequency of never signal. At equilibrium, mean pay-off of always signal equals mean pay-off of never signal. Thus: 


$$
p\left(\frac{1}{2} V-C\right)+(1-p)\left(\frac{3}{4} V-C\right)=p\left(\frac{1}{4} V\right)+(1-p)(0)
$$

Expand terms:

(2) $\frac{1}{2} V p-C p+\frac{3}{4} V-C-\frac{3}{4} V p+C p=\frac{1}{4} V p$

Simplifies to:

(3) $\frac{3}{4} V-C=\frac{1}{2} V p$

Solve for $p$ :

(4) $p=\frac{3 V-4 C}{2 V}$

Given equation 4 , for always signal to be evolutionarily stable and persist in a population $\frac{V}{C}$ must be $>1 \frac{1}{3}$. To get the $\frac{V}{C}$ that results in a pure strategy of always signal, we set $p$ to 1 :

$$
3 V-4 C=2 V, \text { which simplifies to: } V=4 C
$$

Consequently, whenever the benefits to signaling are four or more times the costs of signaling, $p=1$ and the evolutionary stable strategy is a pure always signal strategy. When $\frac{V}{C}$ is between $1 \frac{1}{3}$ and 4 , the evolutionary stable strategy will be a mixed strategy with the equilibrium frequency of the always signal and never signal strategies determined by equation 4 . When $V<4 / 3$, callers are lost, and alternative signaling modalities might be expected to prevail (e.g. cuticular hydrocarbons; Steiger et al. 2013).

\section{Exploring the evolutionary stability of the three strategies}

We explored the evolutionary stability of the three strategies by addressing the following questions: 1) is a population of always signal and never signal individuals at equilibrium vulnerable to invasion by an assess mutant; 2) is a pure population of always signal individuals, or a pure population of never signal individuals, vulnerable to invasion by an assess mutant; 3 ) is a pure population of assess vulnerable to invasion by either an always signal or never signal mutant. This was explored for different values of $V$ and $C$.

1. We begin by considering an evolutionary stable population of caller and satellites in which $V=20$ and $C=10$ (i.e. the benefits of signaling to attract females is twice the cost of signal production). When $V=2 C$, the always signal and never signal strategies have equal fitness.

The equilibrium frequency of always signal individuals can be determined from equation 4 as above: 


$$
\begin{gathered}
p=\frac{3 V-4 C}{2 V} \\
p=\frac{60-40}{40}=0.5
\end{gathered}
$$

Thus, the frequency of always signal individuals is 0.5 and the never signal individuals is $1-p$ or 0.5 . The average payoffs with $V=20$ and $C=10$ are then:

$$
\begin{gathered}
E[\text { always signal }]=p\left(\frac{1}{2} V-C\right)+(1-p)\left(V-C-\frac{1}{4} V\right)=2.5 \\
E[\text { never signal }]=p\left(\frac{1}{4} V\right)=2.5
\end{gathered}
$$

1a. Can this population be invaded by an assess mutant?

When $V=2 C$ half of the encounters involving the assess mutant will be with always signal individuals, and half will be with never signal individuals. The average payoff to the assess mutant is therefore:

$$
E[\text { assess }]=p\left(\frac{1}{4} V\right)+(1-p)\left(V-C-\frac{1}{4} V\right)=5
$$

Thus, when $V=2 C$, assess can invade and spreads in the population.

1b. Can a mutant assess invade a population of pure caller?

Always signal individuals almost exclusively encounter other always signalers, whereas the mutant assess always encounters a signaler. Thus, the average payoff (i.e. fitness) of always signalers and assess when $V=2 C$ is:

$$
\begin{gathered}
E[\text { always signal }]=\frac{1}{2} V-C=0 \\
E[\text { assess }]=\frac{1}{4} V=5
\end{gathered}
$$

Thus, a pure always signal population is vulnerable to invasion by assess.

1c. Can a mutant assess invade a population of pure never signal?

Never signal individuals almost always encounter another never signaler, whereas the mutant assess always encounters a never signaler. The average payoff is therefore:

$$
\begin{gathered}
E[\text { never signal }]=0 \\
E[\text { assess }]=V-C-\frac{1}{4} V=5
\end{gathered}
$$


Thus, assess can invade and spread in a population of pure satellite.

1d. Can a population of pure assess be invaded by either a caller mutant or a satellite $\underline{\text { mutant? }}$

Assess almost always encounters assess, and so too do the mutants. Thus, the average payoffs are:

$$
\begin{gathered}
E[\text { assess }]=\frac{1}{2}(V-C)=5 \\
E[\text { always signal }]=V-C-\frac{1}{4} V=5 \\
E[\text { never signal }]=\frac{1}{4} V=5
\end{gathered}
$$

and at these values of $V$ and $C$ a pure assess population can be invaded by either a caller mutant or a satellite mutant but these mutants are not expected to spread, other than by drift.

2. We next examine when $V \neq 2 C$. Consider the case where $V=30$ and $C=10$. In an evolutionary stable population of always signal and never signal the frequency of always signal (p) will be $\frac{5}{6}$, and the frequency of never signal will be $\frac{1}{6}$ (equation 4 ).

The corresponding payoffs are:

$$
\begin{gathered}
E[\text { always signal }]=p\left(\frac{1}{2} V-C\right)+(1-p)\left(V-C-\frac{1}{4} V\right)=6.25 \\
E[\text { never signal }]=p\left(\frac{1}{4} V\right)=6.25
\end{gathered}
$$

2a. Can this population be invaded by an assess mutant?

If always signal and never signal are in equilibrium, the payoff to assess is:

$$
E[\text { assess }]=p\left(\frac{1}{4} V\right)+(1-p)\left(V-C-\frac{1}{4} V\right)=8 . \overline{33}
$$

Under these conditions of $\mathrm{V}$ and $\mathrm{C}$, assess can invade a population of callers and satellites at equilibrium and will spread.

2b. Assess can invade and spread in a population of pure callers: 


$$
\begin{gathered}
E[\text { always signal }]=\frac{1}{2} V-C=5 \\
E[\text { assess }]=\frac{1}{4} V=7.5
\end{gathered}
$$

2c. Assess can also invade and spread in a population of pure satellites:

$$
\begin{gathered}
E[\text { never signal }]=0 \\
E[\text { assess }]=V-C-\frac{1}{4} V=12.5
\end{gathered}
$$

2d. Can a population of pure assess be invaded by either a caller mutant or a satellite $\underline{\text { mutant? }}$

$$
\begin{gathered}
E[\text { assess }]=\frac{1}{2}(V-C)=10 \\
E[\text { always signal }]=V-C-\frac{1}{4} V=12.5 \\
E[\text { never signal }]=\frac{1}{4} V=7.5
\end{gathered}
$$

At these values of $V$ and $C$, a pure assess population is vulnerable to invasion by an always signal mutant but cannot be invaded by the never signal strategy.

3. Now consider the case where $V=15$ and $C=10$. In a population of always signal and never signal at equilibrium the frequency of always signal $(p)$ will be $\frac{5}{30}$, and the frequency of never signal will be $\frac{25}{30}$.

The corresponding payoffs are:

$$
\begin{gathered}
E[\text { always signal }]=p\left(\frac{1}{2} V-C\right)+(1-p)\left(V-C-\frac{1}{4} V\right)=0.625 \\
E[\text { never signal }]=p\left(\frac{1}{4} V\right)=0.625
\end{gathered}
$$

3a. Can this population be invaded by an assess mutant?

If always signal and never signal are in equilibrium, the payoff to assess is:

$$
E[\text { assess }]=p\left(\frac{1}{4} V\right)+(1-p)\left(V-C-\frac{1}{4} V\right)=1 . \overline{66}
$$

Under these conditions of $V$ and $C$, assess can invade a population of callers and satellites at equilibrium and will spread. 
3b. Assess can also invade a population of pure always signal:

$$
\begin{gathered}
E[\text { always signal }]=\frac{1}{2} V-C=-2.5 \\
E[\text { assess }]=\frac{1}{4} V=1.25
\end{gathered}
$$

3c. Assess can invade a population of pure never signal:

$$
\begin{gathered}
E[\text { never signal }]=0 \\
E[\text { assess }]=V-C-\frac{1}{4} V=1.25
\end{gathered}
$$

3d. Can a population of pure assess be invaded by either a caller mutant or a satellite $\underline{\text { mutant? }}$

$$
\begin{gathered}
E[\text { assess }]=\frac{1}{2}(V-C)=2.5 \\
E[\text { always signal }]=V-C-\frac{1}{4} V=1.25 \\
E[\text { never signal }]=\frac{1}{4} V=3.75
\end{gathered}
$$

So, at these values of $\mathrm{V}$ and $\mathrm{C}$, a pure assess population is vulnerable to invasion by a never signal mutant, but cannot be invaded by the always signal strategy.

\section{Final thoughts and conclusions}

The condition $\mathrm{V}=2 \mathrm{C}$ is an important pivot point for the coexistence of the strategies. At these values of $\mathrm{V}$ and $\mathrm{C}$, all three strategies confer the same fitness, and this seems to be the only condition under which all three strategies can coexist. This pivot point is almost certainly a function of the likelihood that a satellite male will succeed in intercepting a female orienting to a calling male. In our model, we assumed that a satellite male can only intercept females approaching from one of the four cardinal directions, and thus, the payoff to never call in this situation is $\mathrm{V} / 4$. Although this is a reasonable approximation, it remains an open empirical question as to just how successful satellite males are at securing copulations with females orienting to calling males.

When $\mathrm{V}>2 \mathrm{C}$, it seems that that some mixture of callers and assessors is stable, whereas the pure satellite is excluded. When $\mathrm{V}<2 \mathrm{C}$, it seems that some mixture of satellites and assessors is stable, whereas the pure caller is excluded. 
Note that these conclusions appear to hold only when $\mathrm{V} / \mathrm{C}$ lies between 1.33 and 4 . When $\mathrm{V} / \mathrm{C}>4$, the ESS is pure caller, whereas when $\mathrm{V} / \mathrm{C}<1.33$, the ESS is pure satellite.

Empirically, this analysis could provide a powerful diagnostic tool for estimating costs and benefits of calling: whenever we find a population comprising satellites and males that behave something like assessors, it seems likely that $\mathrm{V}<2 \mathrm{C}$; this is what seems to be the case for Gryllodes sigillatus.

\section{Additional References}

Steiger, S., Ower, G.D., Stökl, J., Mitchell, C., Hunt, J. and S.K. Sakaluk. 2013. Sexual selection on cuticular hydrocarbons of male sagebrush crickets in the wild. Proceedings of the Royal Society B 280: 20132353. 
Table S1. Summary of fixed effect estimates on behavioral responses. Results are from an analysis fit using the glmer function of the lme 4 package in $\mathrm{R}$. The clipped treatment was fit as the intercept against which the estimate for the playback treatment was contrasted (i.e. the estimate for Playback would be the sum of the Clipped and Playback estimates). The effects repetition number temperature (Celsius, mean and standard deviation standardized) and mass ( $\mathrm{g}$, mean and standard deviation standardized) on calling propensity and interacted with treatment. Interaction estimates are presented as contrasts versus effects in the Clipped treatment. The model was fit with a binomial error structure.

\begin{tabular}{ccccc}
\hline & Estimate & $\mathrm{SE}$ & $\mathrm{z}$ & $\mathrm{p}$ \\
\hline Intercept (Clipped) & 0.578 & 0.462 & 1.251 & 0.211 \\
vs. Playback & -2.268 & 0.739 & -3.069 & 0.002 \\
\hline Rep (Clipped) & 0.1391 & 0.074 & 1.884 & 0.060 \\
vs. Playback & -0.041 & 0.128 & -0.321 & 0.748 \\
\hline Temperature (Clipped) & 0.0109 & 0.192 & 0.057 & 0.955 \\
vs. Playback & 0.5038 & 0.382 & 1.32 & 0.187 \\
\hline Mass (Clipped) & 0.518 & 0.259 & 2.002 & 0.045 \\
vs. Playback & -0.483 & 0.287 & -1.682 & 0.092 \\
\hline Time (Clipped) & 0.0645 & 0.237 & 0.272 & 0.786 \\
vs. Playback & -0.609 & 0.287 & -2.123 & 0.034 \\
\hline
\end{tabular}

